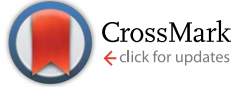

Cite this: Nanoscale, 2014, 6, 9852

\title{
In situ TEM analysis of resistive switching in manganite based thin-film heterostructures $\uparrow$
}

\author{
Jonas Norpoth, Stephanie Mildner, Malte Scherff, Jörg Hoffmann \\ and Christian Jooss*
}

The mechanism of the electric-pulse induced resistance change effect in $\mathrm{Au} / \mathrm{Pr}_{0.65} \mathrm{Ca}_{0.35} \mathrm{MnO}_{3} /$ $\mathrm{SrTi}_{0.99} \mathrm{Nb}_{0.01} \mathrm{O}_{3}$ thin-film samples is studied by means of in situ electrical stimulation inside a transmission electron microscope. A detailed equivalent-circuit model analysis of the measured currentvoltage characteristics provides crucial information for the proper interpretation of the microscopy results. The electrical transport data of the electron-transparent samples used for the in situ investigations is verified by comparison to measurements of unpatterned thin-film samples. We find comprehensive evidence for electrochemical oxygen vacancy migration affecting the potential barrier of the pn junction between $\operatorname{Pr}_{0.65} \mathrm{Ca}_{0.35} \mathrm{MnO}_{3}$ and $\mathrm{SrTi}_{0.99} \mathrm{Nb}_{0.01} \mathrm{O}_{3}$ as well as the resistance of the manganite bulk. The high-resistance state formation in the $\operatorname{Pr}_{0.65} \mathrm{Ca}_{0.35} \mathrm{MnO}_{3}$ bulk is frequently accompanied by structural transformations, namely detwinning and superstructure formation, most likely as the result of the joint impact of dynamic charge inhomogenities by oxygen vacancy migration and injection of high carrier densities at the electrodes.

Received 16th April 2014 Accepted 22nd June 2014

DOI: $10.1039 / c 4 n r 02020 k$

www.rsc.org/nanoscale manifestations are discussed in different frameworks like oxygen vacancy electromigration, ${ }^{\mathbf{1 6 , 1 7}}$ Mott transition, ${ }^{\mathbf{1 4}, 18}$ charge carrier injection and trapping, ${ }^{3,19}$ or current-induced structural phase transitions. ${ }^{20}$ While most of these mechanisms are mainly associated with the interfacial region of the devices, also resistive switching of the $\mathrm{Pr}_{0.7} \mathrm{Ca}_{0.3} \mathrm{MnO}_{3}$ film bulk was directly observed by means of Kelvin probe microscopy. ${ }^{21}$

In this contribution, we address the question of the switching mechanism in $\mathrm{Au} / \mathrm{Pr}_{0.65} \mathrm{Ca}_{0.35} \mathrm{MnO}_{3}$ (PCMO) based thin-film samples by means of in situ transmission electron microscopy (TEM). This technique, where a piezo-driven, metallic nano-tip is used to contact and electrically stimulate an electron-transparent sample inside the microscope, has already been applied to monitor resistive-switching related oxygen vacancy migration in PCMO and other transition-metal oxides, ${ }^{17,22,23}$ as well as corresponding structural phase transitions. ${ }^{20,24-26}$ The high spatial-resolution capabilities of TEM in both imaging and electron-spectroscopy modes allow for indivdual addressing of PCMO bulk, electrodes and interfacial regions respectively in order to detect potential electrically induced changes.

Our PCMO films are epitaxially grown on electron-doped $\mathrm{SrTi}_{0.99} \mathrm{Nb}_{0.01} \mathrm{O}_{3}$ (STNO) substrates forming a $p n$ heterojunction with rectifying transport properties. ${ }^{27,28}$ As will be discussed later by means of an equivalent-circuit model, the contribution of the bottom interface forming the pn junction as well as that of the actual PCMO film and its interface to the Au top electrode can be assigned to different regimes of the current-voltage characteristics. Complementary to the TEM investigations, this feature of our samples provides additional information
Institut für Materialphysik, Universität Göttingen, Friedrich-Hund-Platz 1, 37077 Göttingen, Germany.E-mail: jooss@material.physik.uni-goettingen.de

$\dagger$ Electronic supplementary information (ESI) available. See DOI: $10.1039 / \mathrm{c} 4 \mathrm{nr} 02020 \mathrm{k}$ 
about the resistive switching loci directly from the electrical transport data.

A proper comparison of the in situ transport characteristics acquired from electron-transparent samples (referred to as "TEM-samples" in the following) with data from unpatterned thin-film samples ("pad-samples") is conducted in order to validate the information revealed by the TEM investigations. In this context, the sensitivity of the $p n$ junction current-voltage characteristics on defects and surface states is a useful probe for peculiarities of the TEM-samples, possibly introduced by intrinsic surface effects or surface damage from the sample preparation process, see e.g. ref. 29.

The manuscript is organized as follows: after a description of the experimental details, an equivalent-circuit model is proposed and initially applied to the current-voltage characteristics in a voltage regime below the onset of resistive switching. Afterwards, the EPIR is demonstrated in both types of samples and comparatively analysed. Finally, we present and discuss the results of the in situ TEM investigations.

\section{Experimental procedure}

Epitaxial pn heterojunctions were fabricated by reactive ionbeam sputtering from a stoichiometric PCMO target at a deposition temperature of $750{ }^{\circ} \mathrm{C}$ and an oxygen partial pressure of $10^{-4}$ mbar. Thin films with thickness $d=200-370 \mathrm{~nm}$

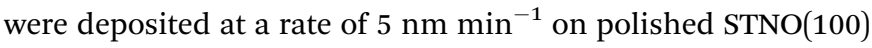
substrates $\left(5 \times 10 \mathrm{~mm}^{2}\right.$; CrysTec). Pre- (post-) deposition heating (cooling) took place at the aforementioned oxygen partial pressure and at a rate of $25{ }^{\circ} \mathrm{C} \mathrm{min}^{-1}$. The electrodes are $\mathrm{Ti}$ contacts with a thin Au protection coating on STNO and Au contacts on PCMO. All metal electrodes were sputter deposited into shadow masks $\left(1 \times 3 \mathrm{~mm}^{2}\right.$ notches $)$ at $110^{\circ} \mathrm{C}$.

In the case of the pad-samples, a permanent photo-resist layer (Allresist AR-U 4040) with lithographically generated circular holes (diameters 3-7 $\mu \mathrm{m}$ ) was placed between PCMO and the shadow mask in order to confine the top electrode contact area to the hole diameter (see Fig. 1(a)).

TEM-samples were prepared by focused ion beam (FIB) technique with a FEI Nova Nano Lab 600. Lamellae of 10-20 $\mu \mathrm{m}$ width were milled as cross-sections of Au/PCMO/STNO samples and glued on $\mathrm{Cu}$ supports by means of chemical vapour deposited $\mathrm{Pt}\left(\mathrm{Pt}^{\mathrm{CVD}}\right)$. The lamellae were then thinned with 30 $\mathrm{keV} \mathrm{Ga}{ }^{+}$ions under grazing incidence of $\alpha=1-2^{\circ}$ and afterwards cleaned with $5 \mathrm{keV} \mathrm{Ga}^{+}$and $\alpha=5^{\circ}$. The final thickness of the various samples covers a range $\Lambda=50-1000 \mathrm{~nm}$ (in electron propagation direction in the TEM). In order to provide well defined cross sections for the electrical characterisation, the lamellae were finally cut into separate, $0.5-2 \mu \mathrm{m}$ wide "teeth" by FIB milling from the top $\left(\alpha=0^{\circ}\right)$. The electrical back contact is provided by the remainder STNO conductively glued to the $\mathrm{Cu}$ support (see Fig. 1(b)).

Electrical characterisation was performed with voltage pulses of $2 \mathrm{~ms}$ duration and $45 \mathrm{~ms}$ period from a programmable Keithley 2430 sourcemeter in two-point configuration. A positive (negative) sign of the applied voltage corresponds to the forward (reverse) direction of the $p n$ junction. One or multiple intermediate pulses at low forward bias were applied between the (incremental) bias pulses in a current-voltage cycle ( $c f$. sketch in Fig. 1(a) of ref. 15). This scheme was implemented in order to monitor potential resistance relaxations after high-power pulses, e.g. as a probe for Joule heat dissipation. In the EPIR regime at high voltages, the intermediate pulses are also useful to determine quasi simultaneously the impact of the resistive switching on the low-bias characteristics, which is rewarding, since the respective regimes of the current-voltage curves are associated with different entities of the equivalent-circuit model (see below). For the TEM investigations, the electrical connection to the TEM-samples was provided by a dedicated in situ TEM holder (Nanofactory) with a piezo-controlled Pt/Ir nano-tip and feed line to the sample support.

The in situ TEM investigations were performed in a Philips CM30 microscope and in a FEI Titan equipped with a GIF Quantum spectrometer, including microscopy techniques like bright field (BF), dark field (DF), annular dark field (ADF) and energy filtered (EFTEM) imaging, selected area diffraction (SAD) and electron energy-loss spectroscopy (EELS). Details of the data analysis for EFTEM and EELS data can be found in the ESI. $\dagger$
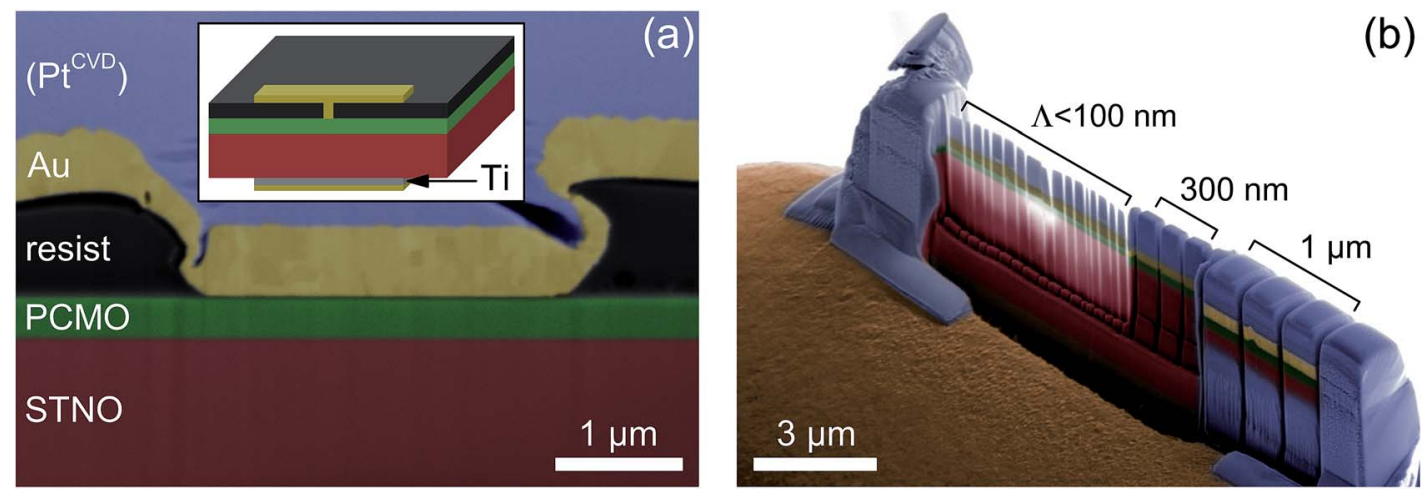

Fig. 1 RGB-colored SEM micrographs of the two types of samples. (a) Cross section through pad-sample. The Pt ${ }^{C V D}$ capping is not present in the active state of the sample. Inset: schematic overview. (b) TEM-sample on Cu support with teeth of different dimensions. 


\section{Electrical transport properties}

\subsection{Rectifying characteristics and equivalent-circuit analysis}

The rectifying current-voltage characteristics of the samples were analysed in terms of numerical simulations of an equivalent-circuit model. This circuit consists of a non-ideal Schottky diode with a reverse bias saturation current $I_{\mathrm{S}}$ and an ideality factor $n$, a series resistance $R_{\mathrm{s}}$ and a parallel shunt resistance $R_{\mathrm{sh}}$ (see inset of Fig. 2(a)). The corresponding current-voltage relation is given by

$$
I(U)=I_{\mathrm{S}}\left[\exp \left(\frac{e\left(U-I R_{\mathrm{s}}\right)}{n k_{\mathrm{B}} T}\right)-1\right]+\frac{U-I R_{\mathrm{s}}}{R_{\mathrm{sh}}},
$$

wherein $e$ and $k_{\mathrm{B}} T$ are the elemental charge and the thermal energy, respectively. Fitting as much as four parameters to experimental current-voltage curves in a consistent manner is only possible because each parameter affects the data in a characteristic way and primarily in a selective voltage interval: the reverse bias region in our samples is entirely dominated by $R_{\mathrm{sh}}$, whereas in forward bias $I_{\mathrm{S}}$ sets the turn-on voltage of the diode, $n$ determines the maximum slope and $R_{\mathrm{S}}$ limits the current at high voltages (see ESI-Fig. $2 \dagger$ ).

In contrast to the geometrically confined TEM-samples, the effective current-carrying cross section in pad-samples is not rigidly defined (see Fig. 1(a)). In particular, there is an asymmetry with respect to the bias polarity, since the primary voltage drop occurs over the diode and the shunt resistance under reverse bias, but over the series resistance in forward direction (see later Fig. 3(d)). Consequently, the (high-bias) forward current scales properly with the top electrode contact area, while it is nearly invariant in reverse direction. Bearing this peculiarity in mind, we normalize the current through the padsamples with the respective top electrode contact areas throughout the following analysis.

Typical diode fit parameters in pad-samples are saturation current densities $j_{\mathrm{s}}=10^{4}$ to $10^{5} \mathrm{Am}^{-2}$ and ideality factors covering a range of $n=2-5$. A closer examination of these numbers will be given below in the context of the TEM-samples.
The series resistance in the pad-samples is dominated by the resistance of the PCMO film and its contact resistance with the $\mathrm{Au}$ electrode. The resistivity of our PCMO films amounts to $\rho_{\text {PCMO }}=1.5 \times 10^{-3} \Omega \mathrm{m}(c f$. also ref. 30$)$. We determined various metal/PCMO contact resistivities by measurements of metal/ $\mathrm{PCMO} /$ metal stack combinations, where we extrapolated from PCMO film thickness series to zero thickness. For Au/PCMO we find a contact resistivity $\delta_{\mathrm{C}}=7.5 \times 10^{-10} \Omega \mathrm{m}^{2}$. We measured the resistivity of STNO to be $\rho_{\mathrm{STNO}}=3 \times 10^{-4} \Omega \mathrm{m}$, but given its large cross section in the samples its contribution to the overall resistance is negligible. We checked both $\mathrm{Ti}$ and $\mathrm{Pt}^{\mathrm{CVD}}$ back electrodes to form ohmic contacts with the STNO and confirmed the ohmic resistivity of $\mathrm{Pt}^{\mathrm{CVD}}$ with $\rho_{\mathrm{pt}: \mathrm{CVD}}=1 \times 10^{-5}$ $\Omega \mathrm{m}$. All numbers are given for room temperature. The major simplification in the model $R_{\mathrm{s}}$ lies in its ohmic character: due to the polaronic nature of its charge carriers, the resistances of both the PCMO and its contact to the Au are known to be current dependent. ${ }^{31}$ However, introducing an extra parameter for the nonlinearity of $R_{\mathrm{S}}$ leads to strong redundance in the data fitting and was thus not eventually implemented in the simulations. Below a current density of $10^{9} \mathrm{Am}^{-2}$ the committed error due to the ohmic approximation should be tolerable, ${ }^{31}$ and we find indeed a satisfying fit accuracy with an ohmic $R_{\mathrm{s}}$ model up to the onset of the EPIR regime: Fig. 2(a) shows typical initial current-voltage curves of pad-samples (all three with $d=370$ $\mathrm{nm}$ and top electrode contact area $\left.A=\pi(3.5 \mu \mathrm{m})^{2}\right)$ and the corresponding model data. Given the above numbers, $R_{\mathrm{S}}=$ $\rho_{\text {PCMO }} \cdot d / A+\delta_{\mathrm{c}} / A=34 \Omega$ is expected, which is in good agreement with the fitted values. The shunt resistance in the pad-samples dominates the reverse bias regime. It is voltage-independent within the measured range and best modeled by an ohmic resistance $R_{\mathrm{sh}} \approx 2500 \Omega$. As stated above, we argue that substantial widening of the effective sample cross section under reverse bias yields such poor rectifying properties. As will be shown in the following paragraph, $R_{\mathrm{sh}}$ is subject to remanent, bipolar resistance changes in the EPIR regime and we thus conclude that its primary origin is of intrinsic nature, i.e. a property of the PCMO/STNO junction and not a processinduced shortage like leakage across the sample edges.
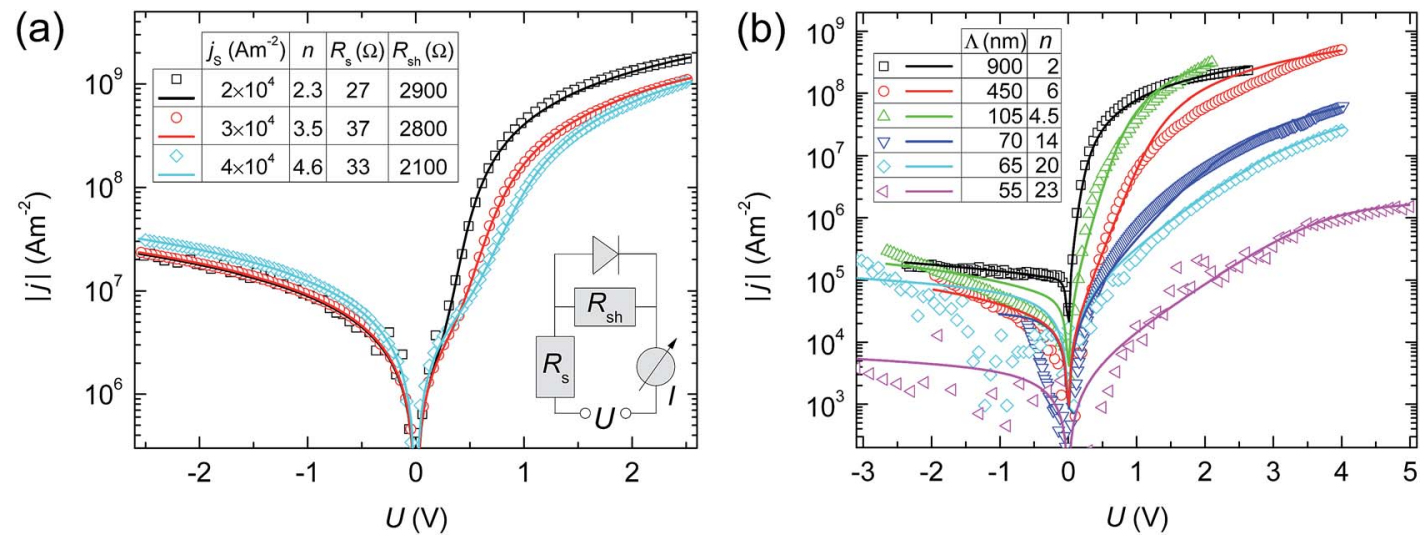

Fig. 2 Initial current-voltage characteristics: experimental (symbols) and simulated (lines) data. (a) Characteristic pad-samples. Top inset: fit parameters. Bottom inset: sketch of the equivalent circuit. (b) Range of TEM-samples. Inset: lamella thickness vs. fitted ideality factor. 
(a)

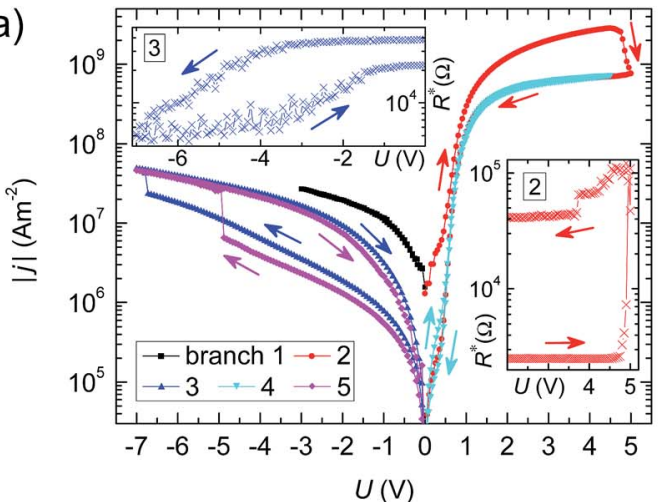

(c)

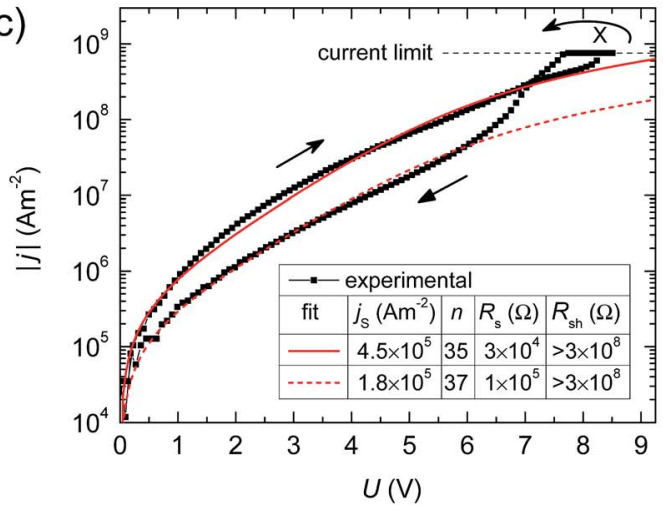

(b)

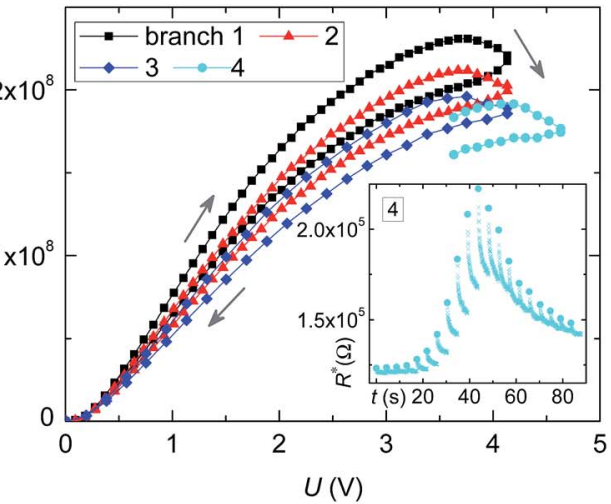

(d)

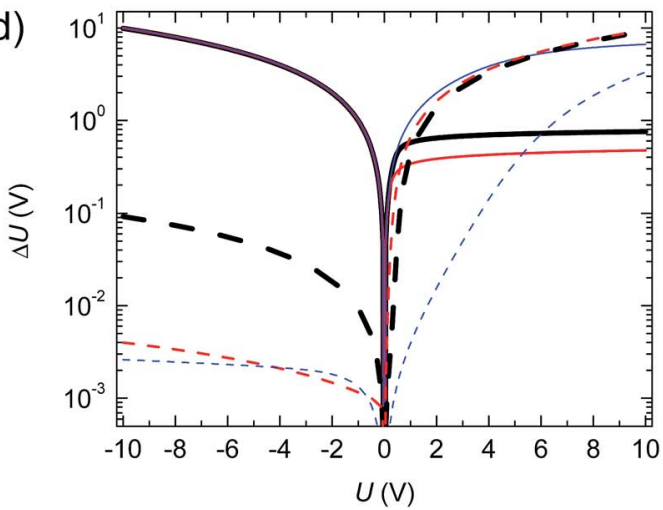

Fig. 3 Electric-pulse induced resistive switching. (a) Pad-sample. Insets: resistance in single intermediate pulses of $0.05 \mathrm{~V}$ forward bias between the incremental pulses in the branches 2 and 3 of the main panel. (b) Thick TEM-sample $(\Lambda=900 \mathrm{~nm})$. Inset: time evolution of the resistance in multiple intermediate pulses of $0.3 \mathrm{~V}$ forward bias after each pulse of branch 4 . (c) Thin TEM-sample $(\Lambda=55 \mathrm{~nm})$. Inset: fit parameters. Arrows indicate the direction of the voltage loops in all viewgraphs. (d) Fractional voltage drop across pn junction (full lines) and series resistance (dashed) for our equivalent-circuit model with parameter sets corresponding to the respective initial current-voltage curves in the subfigures (a)-(c) (thick black, medium red, thin blue).

Microstructural entities possibly acting as shunts are misfit dislocations and boundaries between epitaxial twins in the PCMO film as well as small interfacial $\mathrm{Mn}_{2} \mathrm{O}_{3}$ nanoparticles that we find at an area density of $\sim 1 \mu \mathrm{m}^{-2}$ in TEM/SEM investigations, see ESI-Fig. $3 . \dagger$ These nanoparticles are insulating themselves but may provide conducting channels in their close circumference, e.g. by inversion layer formation..$^{32}$ However, the shunt resistances in TEM-samples exhibit no appreciable differences whether nanoparticles are contained or not. This points to a primary leakage mechanism based on band-to-band-tunneling across the $p n$ junction. ${ }^{33-35}$ Given the high doping levels in both materials, the depletion layer only has a width on the order of 1 (10) nm on the PCMO (STNO) side of the junction. ${ }^{28}$ The corresponding high electric fields across the reverse-biased junction $\left(\sim 10^{8} \mathrm{~V} \mathrm{~m}^{-1}\right.$ at $1 \mathrm{~V}$ applied) give rise to significant current flow by tunneling of electrons from the valence band of the $p$ region into the conduction band of the $n$ region.

Fig. 2(b) shows initial current-voltage characteristics of TEM-samples. There are two striking observations: a strong dependency on the lamella thickness $\Lambda$ and a reduced fitting accuracy with the proposed equivalent-circuit model. In the following we specify the differences between the two types of samples.
With $2<n<6$ and $j_{\mathrm{s}}=10^{4}-5 \times 10^{5} \mathrm{Am}^{-2}$ the fitted diode parameters of thick TEM-samples $(\Lambda>100 \mathrm{~nm})$ closely resemble those of the pad-samples. However, further thinning strongly increases the ideality factor to $n>30$. The ideality factor reflects carrier recombination processes associated with the $p n$ junction: the ideal, defect-free diode with a current exclusively based on diffusion is characterized by $n=1$. The introduction of (independent) traps inside the band gap of the junction gives primarily rise to Shockley-Read-Hall recombination and covers $1<n \leq 2 .{ }^{36,37}$ Higher $n$ values were ascribed to recombination at coupled defects, ${ }^{38}$ tunneling-enhanced recombination at the interface or in the space-charge region, ${ }^{39,40}$ and to multi-step recombination-tunneling. ${ }^{41}$ Brötzmann et al. modeled giant apparent ideality factors by an interfacial disordered or amorphous layer with Frenkel-Poole conduction properties. ${ }^{42}$ We attribute the recombination enhancement in our TEM-samples in part to ion beam induced disorder and defect formation. Binary collision Monte Carlo simulations of the $30 \mathrm{keV}$ FIB milling process show amorphisation of a $5 \mathrm{~nm}$ thick surface layer and an atomic recoil distribution with tails reaching as deep as $15 \mathrm{~nm}$ into the lamellae. ${ }^{43}$ Similar results are reported for FIB-processed TEM samples from $\mathrm{LaAlO}_{3} / \mathrm{SrTiO}_{3}$ multilayers ${ }^{44}$ and even heavier damage formation for $\mathrm{Si}^{45}$ and AlGaAs lamellae. ${ }^{46}$ Since SAD investigations never proved any 
evidence for amorphous material in our samples, we are confident that the final $5 \mathrm{keV}$ cleaning procedure removes the most heavily damaged portion of the surface layer. Nonetheless, the long-reaching recoil tails possibly have an impact on the junction recombination rates. Another reason of the large $n$ values in thin samples might arise from an increased relative contribution of intrinsic surface states. Dong et al. predicted surface reconstructions in low-bandwidth manganites like PCMO that exhibit ferromagnetic-metallic tendencies in contrast to the antiferromagnetic, charge-ordered bulk phase. ${ }^{47}$ Emergent ferromagnetism was indeed observed experimentally in nanoparticles of appropriate manganites. ${ }^{48-50}$ Such metallic surface states could provide additional recombination channels contributing to the modified junction characteristics of our TEM-samples.

The magnitude of the saturation current is only affected below $\Lambda<60 \mathrm{~nm}$, where it eventually declines to the order of $j_{\mathrm{s}} \sim$ $10^{3} \mathrm{Am}^{-2}$.

The series resistance in the measurements of TEM-samples is $2-5$ fold higher than expected based on the values obtained in the pad-samples. We observed that the contact resistance between nano-tip and top electrode yields the dominant extracontribution in our experiments, since repositioning, shifting or pushing the nano-tip in contact with the sample can significantly alter $R_{\mathrm{s}}$. With regard to the EPIR properties, changes in the series resistance of the actual sample can thus only be studied if a low-resistive contact with the nano-tip has been carefully established.

The shunt resistance in TEM-samples exhibits voltage dependence as can be seen in the poor fit accuracy of the reverse branches in Fig. 2(b). A coarse estimate with an ohmic equivalent yields a sheet resistivity on the order of $\delta_{\mathrm{sh}} \sim 10^{-5} \Omega \mathrm{m}^{2}$, which increases up to $\sim 10^{-3} \Omega \mathrm{m}^{2}$ for $\Lambda<60 \mathrm{~nm}$. The nonlinearity, which is most pronounced in the thinnest samples, is presumably also introduced by the surface states providing leakage paths that bypass the pristine band-to-band-tunneling and dominate the reverse current in TEM-samples.

In comparison, the rectifying current-voltage characteristics of pad-samples are basically reproduced in TEM-samples. Surface effects affect the diode parameters of the $p n$ junction in thin lamellae and some experimental care is needed to provide a meaningful $R_{\mathrm{s}}$, which is not dominated by the contact resistance with the nano-tip. The major limitation is the inaccessibility of the pristine PCMO/STNO shunt resistance, which is paralleled by the surface state contributions in TEM-samples.

\subsection{Resistive switching}

Fig. 3(a) shows the typical resistive switching properties of a pad-sample. At a forward bias $U \approx 4 \mathrm{~V}$ and currents $j \gtrsim 3 \times$ $10^{9} \mathrm{Am}^{-2}$, the current-voltage characteristics undergo pronounced remanent changes as quantified by the equivalentcircuit model analysis: $R_{\mathrm{S}}\left(R_{\mathrm{sh}}\right)$ increases by up to a factor of 3 (20), $j_{\mathrm{S}}$ decreases to the order of $10^{3} \mathrm{Am}^{-2}$ while the ideality factor is basically unaffected. Under reverse bias $U \gtrsim 4 \mathrm{~V}, j_{\mathrm{S}}$ increases by a factor of $2-5$, thus only partially recovering its virgin level, and $R_{\mathrm{sh}}$ switches back down though always remaining above its initial value. Reverse biasing does not affect $R_{\mathrm{s}}$ as revealed in the subsequent forward branch (branch 4 in the figure): the high-resistive characteristic at high bias is accurately retraced while only the low-bias region, which is limited by the currents flowing through diode and shunt resistance, exhibits hysteresis. The evolution of the low-bias resistance $R^{*}$ in branch 2 reveals that the changes in the $p n$ junction characteristics emerge simultaneously with the HRS formation in $R_{\mathrm{s}}$. More extensive EPIR data is provided as ESIFig. 4. $\dagger$ Therein, successive current-voltage cycles further increases the low-bias resistance, eventually reaching stable HRS and LRS levels, see (c). After the initial HRS formation in $R_{\mathrm{s}}$, its value remains unaffected by the repeated cycling within the given voltage regime (application of higher voltages soon yields sample failure, see below).

Determining factors for resistive switching are electric field, electric current and Joule heating. In order to estimate the former, we plot the fractional voltage drop across the series resistance $\Delta U\left(R_{\mathrm{S}}\right)=R_{\mathrm{S}} \cdot I$ and across the $p n$ junction $\Delta U($ diode $)=$ $\Delta U\left(R_{\mathrm{sh}}\right)=U-\Delta U\left(R_{\mathrm{s}}\right)$ for our equivalent circuit with different parameter settings in Fig. 3(d). Finite element simulations of the temperature evolution in our pad-samples under electric pulses predict significant local heating only for current densities $j>1 \times 10^{9} \mathrm{Am}^{-2}$, e.g. a temperature increase by $\sim 300 \mathrm{~K}$ at $5 \times 10^{9} \mathrm{Am}^{-2} \cdot{ }^{51}$ This is consistent with our experimental observation of fatal sample failure by crack formation and local melting of the photo-resist layer in this current regime. Altogether, the HRS formation of the series resistance at high forward bias occurs under the combined action of a large voltage drop, high current density and rather massive Joule heating. Under reverse bias neither of these quantities acts comparably strong on $R_{\mathrm{s}}$, which consequently remains unchanged. In contrast, reverse biasing yields a nearly complete voltage drop across the $p n$ junction. The emerging electric fields (on the order of $\sim 10^{8} \mathrm{Vm}^{-1}$ as stated above) are apparently sufficient to counteract the foregoing forward bias switch and partially recover $j_{\mathrm{S}}$ and $R_{\mathrm{sh}}$ towards their initial values.

The low-bias resistance in between the "pump" pulses displays temporal relaxation behaviour on both forward and reverse bias branches (see insets in Fig. 3(a)). Based on the respective current density levels, only the relaxation on the forward branch can be assigned to Joule heat dissipation, while no significant temperature increase is expected under reverse bias. As will be discussed later, we interprete this finding as evidence for relaxation of an oxygen vacancy distribution driven out of equilibrium.

Given the aforementioned limitations imposed by the surface contributions in TEM-samples, the in situ TEM investigations of the EPIR are restricted to the effects accompanying the HRS formation in $R_{\mathrm{s}}$. Especially, changes of the $p n$ junction shunt resistance can hardly be observed, since the reverse bias branch as well as the low-bias regime of the forward branch are

† We performed systematic measurements with multiple low-bias pulses following "pump" forward bias pulses. Resistance relaxation effects were only found after pump pulses with $j \geq 1 \times 10^{9} \mathrm{Am}^{-2}$. They are ascribed to Joule heating, consistent with the results of the finite element simulations. 
dominated by the parasitic contributions of the surface states. Although we are thus restricted to the analysis of the forward bias branches, we verified that reverse biasing does not affect $R_{\mathrm{s}}$, consistent with the findings in pad-samples. Furthermore, the differences in thermal coupling, i.e. the much lower heat conductivity compared to pad-samples due to the surfaces with vacuum, lowers the threshold current for TEM-sample failure by approximately one order of magnitude to $j \geq 5 \times 10^{8} \mathrm{Am}^{-2}$.

Fig. 3(b) shows the HRS formation in a thick TEM-sample. The successive forward biasing eventually increases $R_{\mathrm{S}}$ by a factor of $\sim 2$ compared to the initial state. The TEM-sample has a low ideality factor $n \approx 2$ and therefore the fractional voltage drop across its equivalent-circuit components corresponds to the relations in pad-samples (see Fig. 3(d)). The relative increase of $R_{\mathrm{s}}$ as well as the switching voltage $U \approx 4 \mathrm{~V}$ closely resemble the observations in pad-samples. However, the current density during resistive switching is about one order of magnitude lower. Given the reduced heat conductivity of the TEM-samples, this finding suggests that Joule heating plays a major role for the HRS formation. The relaxation of the lowbias resistance in the inset of Fig. 3(b) follows an exponential decay $R^{*}(t) \propto \exp (-t / \tau)$ with $\tau \approx 1 \mathrm{~s}$, which equals the corresponding time constants found for pad-samples (not shown). Likewise, we consider this relaxation behaviour in the EPIR regime in part as due to Joule heat dissipation, but possibly also as the fingerprint of the equilibration of a highly driven state, which is characterized by the onset of oxygen vacancy electromigration and unbalanced charge carrier exchange rates (see below). $\S$

Fig. 3(c) shows resistive switching in a thin TEM-sample, whose high ideality factor allows for proper fitting of changes in the diode parameters. Due to the lower fractional voltage drop across the series resistance, the current respectively heating level for switching is only reached at $U>8 \mathrm{~V}$. Irregular behaviour at high forward bias, as displayed in this viewgraph, is occasionally observed within the narrow current regime between switching and sample failure ( $c f$. section 4$)$.

In comparison with pad-samples, the HRS formation in $R_{\mathrm{S}}$ is well reproduced in TEM-samples. In both types of samples, $R_{\mathrm{S}}$ increases at sufficient forward bias by a factor of up to 3 , while it is unaffected by reverse biasing. The current density during switching is in each case close to the respective samplefailure threshold indicating the presence of similar levels of Joule heating. Concerning the equivalent-circuit model parameters of the very $p n$ junction, only the initial decrease of $I_{\mathrm{S}}$ accompanying the HRS formation in $R_{\mathrm{S}}$ is evident in TEMsamples, while switching of the intrinsic $R_{\mathrm{sh}}$ as well as the rather subtle changes associated with repeated reverse biasing remain hidden behind the parasitic contributions of the surface states.

\footnotetext{
$\S$ The $R^{*}$ in Fig. 3(b) is measured at a higher forward bias than in (a) since the low-bias regime in the TEM-samples is dominated by the parasitic surface contributions. The interpretation of $R^{*}$ on the basis of the equivalent-circuit model is therefore not equally straightforward and has to deal not only with parameters related to the $p n$ junction $\left(I_{\mathrm{S}}, R_{\mathrm{sh}}\right)$ but also with $R_{\mathrm{S}}$.
}

\section{Microstructural changes accompanying resistive switching}

The TEM investigations of microstructural changes during resistive switching are capable of addressing the different elements of the equivalent-circuit individually, i.e. the interfacial region around the $p n$ junction, governing the diode characteristics and $R_{\mathrm{sh}}$, and the PCMO film and its interface to the Au electrode jointly constituting $R_{\mathrm{S}}$.

Fig. 4 shows the results of EFTEM/EELS investigations of the oxygen $\mathrm{K}$ edge before and after electric-pulse induced resistive switching of a TEM-sample. The electrical stimulation with a pulsed forward bias loop led to HRS formation, namely a doubling of $R_{\mathrm{S}}$ and a decrease of $j_{\mathrm{S}}$ by a factor of $\sim 5$ as determined by the fits (see inset of (c)). Despite the low signal-tonoise ratio in the EFTEM data, subfigures (a)-(c) reveal a subtle but regularly observed peculiarity in the elemental oxygen distribution, namely an oxidation of the STNO by high forward currents in an initially oxygen-depleted region below the interface to the PCMO film, extending $\sim 50 \mathrm{~nm}$ into the substrate (shaded area in (c); see ESI-Fig. $4 \dagger$ for another example). Although Fig. 4(c) may indicate an apparent global oxygen uptake, this was not systematically observed throughout our experiments, in particular not for the PCMO film area. Furthermore, EFTEM elemental maps of the cations are unaffected by electrical stimulation.

The observation of an oxygen-deficient STNO surface zone in pristine TEM-samples is rather surprising, since oxygen concentration-cell experiments suggest equilibrium vacancy concentrations only on the order of $10^{19}-10^{20} \mathrm{~cm}^{-3}$ under our deposition conditions. ${ }^{52}$ Additional vacancies are possibly introduced by gettering effects of the PCMO overlayer, ${ }^{53-55}$ or by the irradiation during FIB processing.

Nevertheless, the observed electromigration of vacancies under forward biasing is reasonable on the basis of diffusive transport data reported in the literature. For the diffusion of (preexisting) oxygen vacancies in STO single crystals, an activation energy $E_{\mathrm{A}}=0.26 \mathrm{eV}$ and a diffusion constant $D_{0}=1.3 \times$ $10^{-5} \mathrm{~cm}^{2} \mathrm{~s}^{-1}$ are reported, ${ }^{56}$ consistent with a diffusion coefficient $D(T=500 \mathrm{~K}) \approx 2 \times 10^{-8} \mathrm{~cm}^{2} \mathrm{~s}^{-1}$ in ref. 57 . According to the Huntington-Grone theory, ${ }^{58}$ the vacancy drift velocity in the presence of an applied electric field $E$ equals $v=D Z^{*} e E / k_{\mathrm{B}} T$, wherein we set the effective charge of the oxygen vacancies to $Z^{*}=+2$. For the parameter regime of the resistive switching in the TEM-samples, i.e. $T=400-600 \mathrm{~K}$ and $E=j \cdot \rho_{\text {STNO }}$ with $j=2 \times$ $10^{8} \mathrm{Am}^{-2}$ and $\rho_{\text {STNO }}(T)=(0.5-1) \times 10^{-3} \Omega \mathrm{m}$, this yields a coarse estimate of the oxygen vacancy diffusion lengths $\Delta x=t_{\mathrm{p}} v \approx 10$ $100 \mathrm{~nm}$ within our electric pulses of $t_{\mathrm{p}}=2 \mathrm{~ms}$ duration.

The analysis of the electron-loss near-edge structure (ELNES) of the oxygen K edge in Fig. 4(d) provides detailed information about the electronic structure of the $p n$ junction and its changes by the electrical stimulation. The $\mathrm{O}$ K edge $(1 \mathrm{~s} \rightarrow 2 \mathrm{p}$ transition) probes the unoccupied states above the Fermi level and can be interpreted as the site- and symmetry-projected density-ofstates given the strong screening of the remaining core-hole. ${ }^{59}$ The first ELNES feature (labelled $K_{1}$ ) arises from covalent 

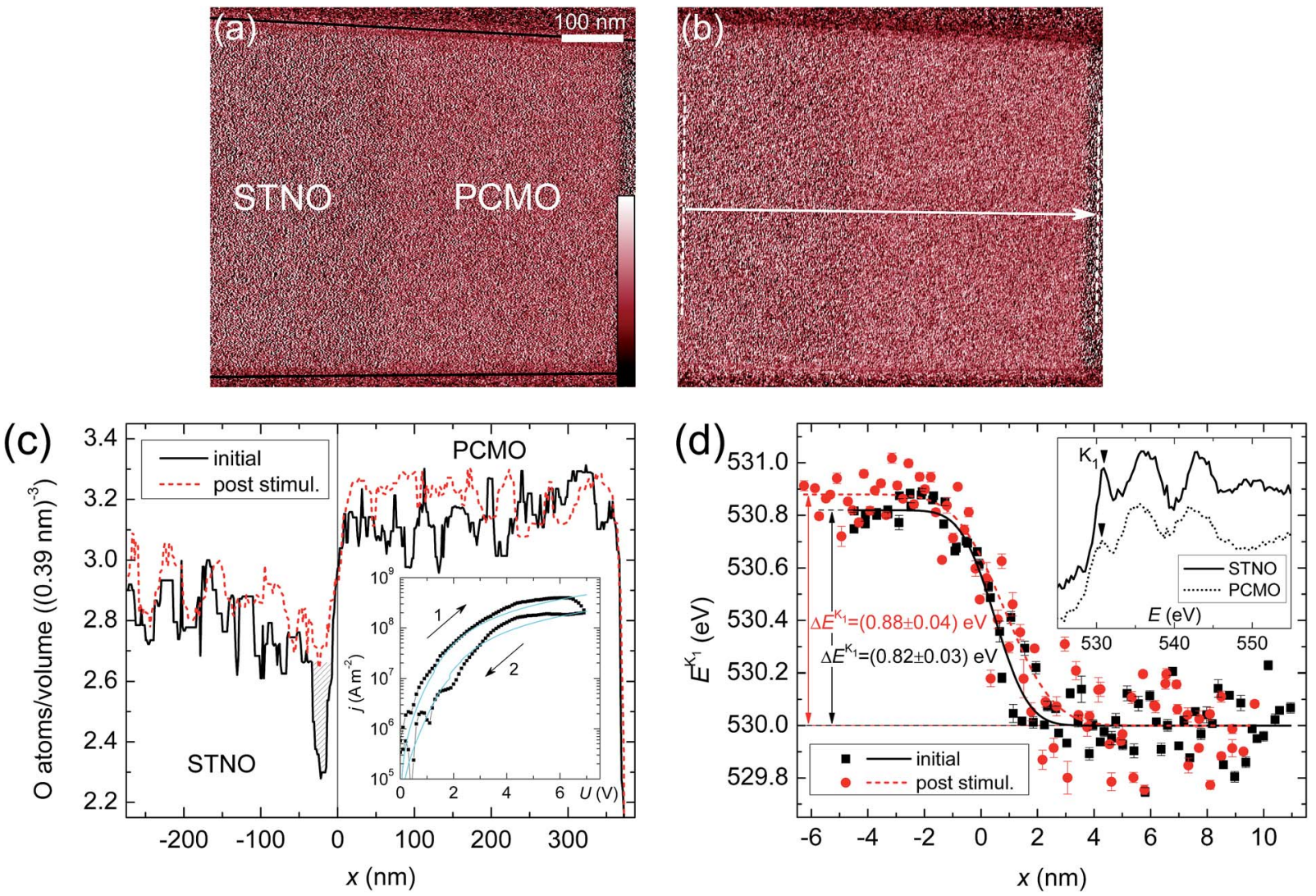

Fig. 4 Elemental oxygen maps and O K ELNES evolution across the PCMO/STNO junction. False-color maps of atomic volume density from EFTEM (a) before and (b) after electric-pulse induced HRS formation. The black lines in (a) mark the TEM-sample contour. (c) Averaged line profiles from areas as indicated in (b), $x=0$ corresponds to the position of the junction. The profiles are additionally smoothed by a median filter of $15 \mathrm{~nm}$ width. (See ESI† for a comment on the accuracy of the absolute values). Inset: in situ current-voltage curve (symbols) and corresponding fits (lines); the map in (b) was acquired after this stimulus. Fit parameters on branch $1(2)$ are $j_{\mathrm{S}}=5(1) \times 10^{5} \mathrm{Am}{ }^{-2}, n=13(13), R_{\mathrm{s}}=2.5(5) \times$ $10^{5} \Omega$ and $R_{\mathrm{sh}} \approx 1(1) \times 10^{8} \Omega$. (d) Evolution of the peak center of OK ELNES feature $K_{1}$ across the junction. Symbols are determined from individual spectra in line scans (see ESI + ), lines are corresponding error function fits. Data is offset to a joint energy level in PCMO. Inset: O K ELNES in the bulk materials.

mixing of $\mathrm{O} 2 \mathrm{p}$ with the transition metal $3 \mathrm{~d}$ states and represents the bottom of the respective conduction bands. ${ }^{60-62}$ The conduction band offset $\Delta E^{\mathrm{K}_{1}}$ across the junction determines the reverse barrier $\phi_{\mathrm{b}}$ for electrons from the PCMO side carrying the reverse saturation current, $I_{\mathrm{S}} \propto \exp \left(-\phi_{\mathrm{b}} / k_{\mathrm{B}} T\right)$. The slight increase $\Delta \phi_{\mathrm{b}} \approx \Delta E_{\mathrm{HRS}}^{\mathrm{K}_{1}}-\Delta E_{0}^{\mathrm{K}_{1}}=0.06 \mathrm{eV}$ in the HRS after oxidation of the STNO interfacial region by the electrical stimulation corresponds to a concomitant decrease of the saturation current $I_{\mathrm{S}, \mathrm{HRS}} / I_{\mathrm{S}, 0}=\exp \left(-\Delta \phi_{\mathrm{b}} / k_{\mathrm{B}} T\right) \approx 0.1$ at room temperature. This value is in line with the regularly observed switch of $I_{\mathrm{S}}$ by an order of magnitude in the current-voltage curves of both TEM- and pad-samples. Likewise, an increase of $\phi_{\mathrm{b}}$ by oxidation of the $\mathrm{SrTiO}_{3}$ interfacial region was also predicted by Jeon et al. for Schottky junctions of $\mathrm{SrTiO}_{3}$ with $\mathrm{SrRuO}_{3} \cdot{ }^{63}$

The reduction of the $n$-doping level in the interfacial STNO by the loss of oxygen vacancies also accounts for the increase of $R_{\mathrm{sh}}$ observed in pad-samples: due to the corresponding widening of the depletion layer, the underlying band-to-bandtunneling across the junction suffers from a reduction of the built-in electric field (or an increase of the effective tunneling length respectively).
Reverse biasing of TEM-samples leaves no visible effects in EFTEM elemental maps or EELS spectra. Given the low current level in the reverse regime, any change in the oxygen vacancy distribution accompanying the partial recovery of $I_{\mathrm{S}}$ and $R_{\mathrm{sh}}$ towards their initial values is supposed to take place in the narrow region of high electric field around the junction, but cannot be resolved in our experiments. At least, we argue that the resistance creep observed in the backward scan $\left(-U_{\max } \rightarrow\right.$ 0 ) of reverse bias branches in pad-samples (see top inset of Fig. 3(a)) is an indication of a slow oxygen vacancy redistribution.

The HRS formation in $R_{\mathrm{s}}$ is not reflected by any spectroscopically detected changes at the interface between PCMO and $\mathrm{Au}$ top electrode. The very interface consists of a $\sim 2 \mathrm{~nm}$ wide amorphous PCMO layer with an increased oxygen/cation atomic ratio of $\sim 3$ compared to $3 / 2$ in bulk PCMO (see ESI-Fig. $5 \dagger$ ). Width and stoichiometry of this layer as well as $\mathrm{O} \mathrm{K}$ and $\mathrm{Mn} \mathrm{L}$ ELNES in the interfacial region are unaffected by both forward and reverse biasing. We find no evidence for an electrochemical redox mechanism involving the Au top electrode as proposed for the resistive switching of interfaces between PCMO and 
various less noble metal electrodes. ${ }^{\mathbf{1 0 , 1 1 , 1 3 , 6 4}}$ The anodic oxidation of the Au electrode under forward bias is supposed to be energetically hindered by the high standard enthalpy of formation for Au-oxide $\left(\Delta H_{\mathrm{f}}^{0}=-13 \mathrm{~kJ} \mathrm{~mol}^{-1}\right.$ or even > $+100 \mathrm{~kJ} \mathrm{~mol}^{-1}$ for $\mathrm{Au}_{2} \mathrm{O}_{3},{ }^{65,66}$ compared to $-80 \mathrm{~kJ} \mathrm{~mol}^{-1}$ for $\mathrm{PtO}_{2},{ }^{67}$ and the oxides of less noble metals widely discussed for the formation of interfacial oxide layers, e.g. $\mathrm{WO}_{2}$ $-590 \mathrm{~kJ} \mathrm{~mol}^{-1}, \mathrm{TiO}_{2}-945 \mathrm{~kJ} \mathrm{~mol}^{-1}$ or $\mathrm{Al}_{2} \mathrm{O}_{3}-1676 \mathrm{~kJ} \mathrm{~mol}^{-1}$ (ref. 68)). Under reverse bias the driving force for oxygen migration out of the interfacial region is insignificant given the minor fractional voltage drop across the PCMO/Au interface (remind of Fig. 3(d)). Consistent with our findings, also Li et al. and Kim et al. report no evidence for anodic oxidation of $\mathrm{Au}$ electrodes in the switching regime of PCMO-based samples. ${ }^{\mathbf{1 4 , 6 4}}$

The HRS formation in $R_{\mathrm{S}}$ is therefore ascribed to changes within the PCMO film. Resistive switching of bulk PCMO in addition to interface effects was also reported from scanning Kelvin probe measurements of a switching device between laterally arranged electrodes. ${ }^{21}$ As stated above, the oxygen distributions in the PCMO films as revealed in our EFTEM experiments exhibit no systematic changes by the electrical stimulation. Literature data for oxygen vacancy diffusion in hole-doped manganites yield higher activation energies compared to STO, namely $E_{\mathrm{A}}=0.4-1.3 \mathrm{eV} .^{\mathbf{1 6 , 6 9 , 7 0}}$ Due to the corresponding shorter diffusion length as well as the lower vacancy concentration compared to the oxygen-depleted STNO surface layer, any electromigrative redistribution is supposed to take place below the EFTEM detection limit. However, evidence for a major contribution of oxygen vacancy migration to the HRS formation in $R_{\mathrm{s}}$ is provided by the electrical transport data. Comparing current-voltage curves and intermediate pulses, we always observe that the HRS formation in both $R_{\mathrm{s}}$ and the $p n$ junction occurs simultaneously - see e.g. branch 2 in Fig. 3(a), where the current drop at $U>4.5 \mathrm{~V}$, representing the HRS formation in $R_{\mathrm{s}}$, is accompanied by a simultaneous increase of the low-bias resistance, which is entirely dominated by the junction parameters (remind of ESI-Fig. $2 \dagger$ ) and caused by the electromigrative oxidation of the STNO side of the junction as shown in the previous paragraph. We attribute this coincidence to a global temperature increase by Joule heating crossing a threshold value for thermally activated electromigration of oxygen vacancies. The concentration of oxygen vacancies is a key parameter controlling the transport properties of manganites, especially since they act as electron donors and thus significantly affect the delicate balance between itineracy and localization tendencies in Mn 3d states. It was recently shown, e.g., that oxygen vacancies in PCMO can induce local Mott gaps, mimicking the electronic structure of the undoped, insulating parent compound $\mathrm{PrMnO}_{3} .{ }^{18}$ Taking into account the qualitative picture of these correlation physics by a double-exchange Hamiltonian, Quintero et al. found indeed that subtle oxygen vacancy redistributions between bulk and interfacial region can account for the resistive switching properties observed in $\mathrm{Pr}_{0.3} \mathrm{La}_{0.075} \mathrm{Ca}_{0.325} \mathrm{MnO}_{3}$ films. ${ }^{72}$ An in situ TEM observation of

I The vacancy formation enthalpy has been subtracted from the self-diffusion data in ref. 70 based on the data provided in ref. 71 . oxygen vacancy migration in PCMO under a steady electric field of $\sim 9 \times 10^{6} \mathrm{Vm}^{-1}$ was reported by Liao et al. ${ }^{17}$ This value corresponds to the field strength across the PCMO films applied in the EPIR regime of our samples.\|

Another source for doping level modulations are the differences of the charge carrier mobilities in the involved materials, namely small hole polarons with thermally activated hopping transport properties in PCMO, ${ }^{73}$ and (quasi-)free conduction band electrons in STNO and Au, respectively. It has been pointed out by Emin that the electrical transport under such circumstances is governed by the low intersite hopping rate of the small polarons and especially by the supposedly even lower rate of conversion between quasifree and polaron carriers at the interfaces to the electrodes. ${ }^{74}$ Likewise, the domain model of Rozenberg et al. with two distinguished carrier transfer rates between electrode and interface domains as well as between interface and bulk domains captures the salient features of bipolar, nonvolatile memory devices. ${ }^{75,76}$ Emin predicted ibid. that a driven non-equilibrium state with a strongly inhomogeneous polaron density could result in a dynamic destabilization of the polarons into a highly conductive state that in turn couples to the lattice and eventually induces a structural phase transition. Our in situ TEM measurements indeed yield experimental evidence for such a process during the HRS formation, see Fig. 5. Therein, the in situ recorded SAD patterns reveal a dynamic change of the PCMO twin system, namely a transformation of the initial [100] zone axis in subfigure (a) to a dominant [010] orientation with only minor remnants of the pristine twin in (b). The transition between the twins equals subtle tiltings of the $\mathrm{MnO}_{6}$ octahedra within the orthorhombic Pbnm structure (space group no. 62). ${ }^{77}$ The vibrational patterns of the $\mathrm{MnO}_{6}$ octahedra constitute the relevant phonon modes of the small polaron formation in PCMO. ${ }^{78-80}$ Highly driven polaron states can thus give rise to strong excitations of the octahedral tilt system, which in combination with Joule heating can induce changes in the twin system. We observe this transformation frequently under high forward currents on the order of $10^{8} \mathrm{Am}^{-2}$, i.e. in the EPIR regime. The highly irregular shape of the corresponding current-voltage curve in Fig. 3(c) with an intermediately augmented conductivity is a rather extreme example of the nonlinear characteristics that sometimes, but not always, accompany the twin transformation. Such irregularities are also found in pad-samples, but only at one-order-ofmagnitude higher current densities, which indicates that Joule heating is an important contributor (remind of the different heat conductivities of TEM- and pad-samples).

In addition to the frequently observed twin transformation, the HRS formation is sometimes accompanied by a further structural phase transition as displayed in Fig. 5(c) (see ESIFig. $6 \dagger$ for another example). Based on the TEM investigations, this structure is reminiscent to the charge- and orbital-ordered,

|| The reported slow vacancy drift velocity ( $\sim 10 \mathrm{~nm}$ in $100 \mathrm{~s})$ seems to be not in line with the ms-timescale of our voltage-pulse experiments. We argue that this discrepancy is caused by different levels of Joule heating, since the electric current density during the stimulation is approximately two orders of magnitude higher in our experiments. 


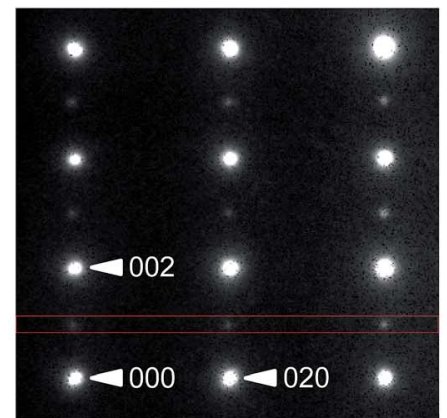

(a)

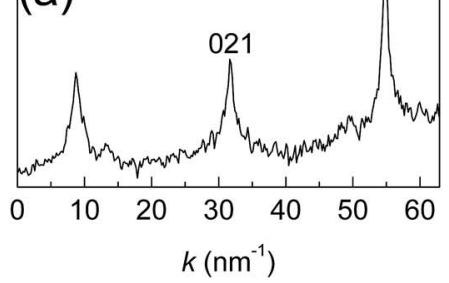

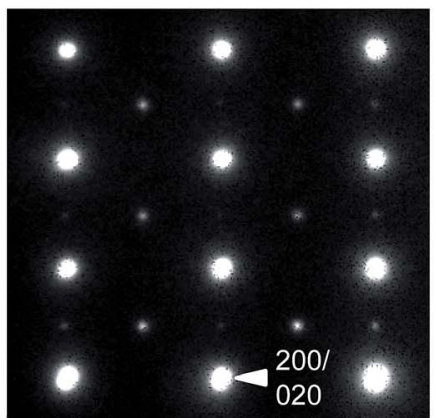

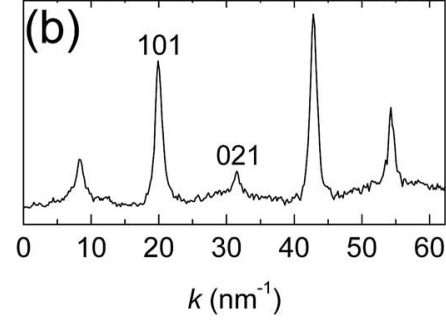

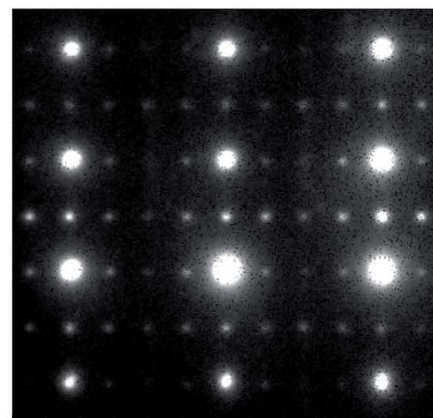

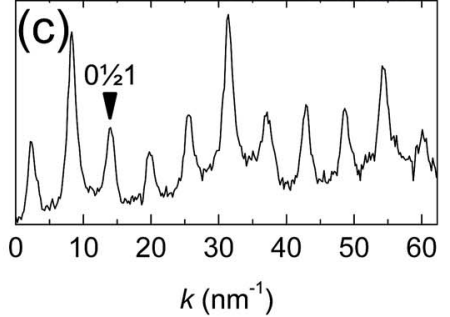

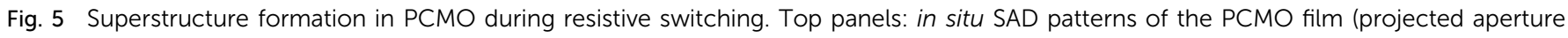

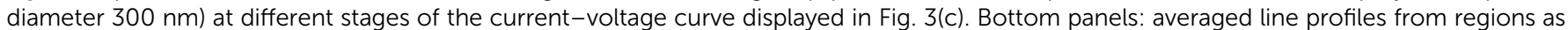

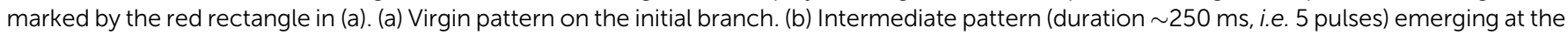
onset of resistive switching marked by the cross in Fig. 3(c). (c) Persistent final pattern on the backward branch following the pattern in (b).

low-temperature $P 2{ }_{1} n m$ phase of PCMO (space group no. 31) with a real-space doubling of the unit cell in the crystallographic $b$-direction. ${ }^{78}$ We already reported such electrically induced superstructure formation for PCMO bulk samples. ${ }^{24}$ In TEMsamples, superstructure domains are found to nucleate at both interfaces to STNO and Au respectively and sometimes even spread across the whole PCMO film in a filamentary manner. The $P 2_{1} \mathrm{~nm}$ phase is characterized by the long-range ordering of Zener polarons on specific lattice sites with a nominal density of 0.5 holes per pseudo-cubic unit cell, which is higher than the average density in PCMO with $x=0.35$ Ca doping. We thus argue that the electrically induced superstructure formation is a means of accommodating an inhomogeneous carrier density, which occurs in the EPIR regime by the combined impact of oxygen vacancy electromigration and high-mobility carrier injection/extraction at the electrodes.

\section{Conclusions}

Resistive switching in Au/PCMO/STNO thin-film heterostructures was studied by means of in situ TEM and equivalentcircuit model analysis. The electrical transport properties of both electron-transparent cross-section samples used for in situ TEM and unpatterned reference samples prove to be basically identical, namely rectifying current-voltage characteristics due to the PCMO/STNO pn junction with forward currents being limited by the series resistance of the manganite film and its interface to the $\mathrm{Au}$ top electrode. Under sufficient forward currents and a corresponding Joule heating level, a nonvolatile high-resistance state is formed in the PCMO film evident as an increase of the series resistance. Simultaneously, the reverse saturation current across the $p n$ junction as well as its shunt resistance decreases and increases, respectively. The built-in asymmetry of the current-voltage characteristics with respect to the voltage polarity entails that only these junction related resistive switching effects can be reversed under reverse bias, since the only action in this regime is imparted by the voltage drop across the pn junction. The in situ TEM investigations revealed that the resistive switching of the $p n$ junction under forward bias is accompanied by an electromigrative oxidation of an initially oxygen-depleted surface layer in the STNO, gradually increasing the height of the junction potential barrier which represents the underlying reason for the change of the junction parameters. In contrast, the high-resistance state formation in the series resistance is not reflected by microstructural or chemical changes of the interface with the top electrode, in particular, no anodic oxidation of the Au was observed. Instead, we frequently observed a detwinning of the epitaxial PCMO microstructure as well as a structural transition to a long-range ordered phase reminiscent of the insulating ground state of PCMO. These findings indicate that changes within the bulk PCMO film contribute to the resistive switching, in contrast to the interface-dominated switching in devices with non-noble metal electrodes. We argue that the fundamental impetus underlying these modifications stems from electronic inhomogenities, which are imposed by electromigrative oxygen vacancy redistribution as well as by non-equilibrium charge carrier exchange rates between PCMO and the electrodes.

\section{Acknowledgements}

We thank H. Hofsäss (II. Phys. Institut, Universität Göttingen) for the MC simulations of the irradiation damages induced by FIB sample preparation. Funding by the Deutsche Forschungsgemeinschaft (DFG) under grant numbers JO 348/10-1 and SFB1073 is acknowledged. 


\section{References}

1 S. Q. Liu, N. J. Wu and A. Ignatiev, Appl. Phys. Lett., 2000, 76, 2749.

2 A. Sawa, Mater. Today, 2008, 11, 28.

3 Y. Chen, L. Chen, G. Lian and G. Xiong, J. Appl. Phys., 2009, 106, 023708.

4 W. W. Zhuang, W. Pan, B. D. Ulrich, J. J. Lee, L. Stecker, A. Burmaster, D. R. Evans, S. T. Hsu, M. Tajiri, A. Shimaoka, K. Inoue, T. Naka, N. Awaya, A. Sakiyama, Y. Wang, S. Q. Liu, N. J. Wu and A. Ignatiev, Int. Electron Devices Meet., 2002, 193.

5 R. Waser, R. Dittmann, G. Staikov and K. Szot, Adv. Mater., 2009, 21, 2632.

6 H. Akinaga and H. Shima, Proc. IEEE, 2010, 98, 2237.

7 Y. Fujisaki, Jpn. J. Appl. Phys., 2013, 52, 040001.

8 Z. Liu, P. Zhang, Y. Meng, H. Tian, J. Li, X. Pan, X. Liang, D. Chen and H. Zhao, Appl. Phys. Lett., 2012, 100, 143506.

9 K. Tsubouchi, I. Ohkubo, H. Kumigashira, M. Oshima, Y. Matsumoto, K. Itaka, T. Ohnishi, M. Lippmaa and H. Koinuma, Adv. Mater., 2007, 19, 1711.

10 S. Asanuma, H. Akoh, H. Yamada and A. Sawa, Phys. Rev. B: Condens. Matter Mater. Phys., 2009, 80, 235113.

11 K. Shono, H. Kawano, T. Yokota and M. Gomi, Applied Physics Express, 2008, 1, 055002.

12 Z. L. Liao, Z. Z. Wang, Y. Meng, Z. Y. Liu, P. Gao, J. L. Gang, H. W. Zhao, X. J. Liang, X. D. Bai and D. M. Chen, Appl. Phys. Lett., 2009, 94, 253503.

13 F. Borgatti, C. Park, A. Herpers, F. Offi, R. Egoavil, Y. Yamashita, A. Yang, M. Kobata, K. Kobayashi, J. Verbeeck, G. Panaccione and R. Dittmann, Nanoscale, 2013, 5, 3954.

14 D. S. Kim, Y. H. Kim, C. E. Lee and Y. T. Kim, Phys. Rev. B: Condens. Matter Mater. Phys., 2006, 74, 174430.

15 M. Scherff, B.-U. Meyer, J. Hoffmann and C. Jooss, J. Appl. Phys., 2011, 110, 043718.

16 Y. B. Nian, J. Strozier, N. J. Wu, X. Chen and A. Ignatiev, Phys. Rev. Lett., 2007, 98, 146403.

17 Z. Liao, P. Gao, X. Bai, D. Chen and J. Zhang, J. Appl. Phys., 2012, 111, 114506.

18 H. S. Lee, S. G. Choi, H.-H. Park and M. J. Rozenberg, Sci. Rep., 2013, 3, 1704.

19 S. T. Hsu, T. Li and N. Awaya, J. Appl. Phys., 2007, 101, 024517.

20 C. Jooss, J. Hoffmann, J. Fladerer, M. Ehrhardt, T. Beetz, L. Wu and Y. Zhu, Phys. Rev. B: Condens. Matter Mater. Phys., 2008, 77, 132409.

21 X. Chen, N. J. Wu, J. Strozier and A. Ignatiev, Appl. Phys. Lett., 2005, 87, 233506.

22 P. Gao, Z. Wang, W. Fu, Z. Liao, K. Liu, W. Wang, X. Bai and E. Wang, Micron, 2010, 41, 301.

23 S.-J. Choi, G.-S. Park, K.-H. Kim, W.-Y. Yang, H.-J. Bae, K.-J. Lee, H. i. Lee, S. Y. Park, S. Heo, H.-J. Shin, S. Lee and S. Cho, J. Appl. Phys., 2011, 110, 056106.

24 C. Jooss, L. Wu, T. Beetz, R. F. Klie, M. Beleggia, M. A. Schofield, S. Schramm, J. Hoffmann and Y. Zhu, Proc. Natl. Acad. Sci. U. S. A., 2007, 104, 13597.
25 L. J. Zeng, H. X. Yang, Y. Zhang, H. F. Tian, C. Ma, Y. B. Qin, Y. G. Zhao and J. Q. Li, Europhys. Lett., 2008, 84, 57011.

26 D.-H. Kwon, K. M. Kim, J. H. Jang, J. M. Jeon, M. H. Lee, G. H. Kim, X.-S. Li, G.-S. Park, B. Lee, S. Han, M. Kim and C. S. Hwang, Nat. Nanotechnol., 2010, 5, 148.

27 A. Sawa, T. Fujii, M. Kawasaki and Y. Tokura, Appl. Phys. Lett., 2005, 86, 112508.

28 G. Saucke, J. Norpoth, C. Jooss, D. Su and Y. Zhu, Phys. Rev. B: Condens. Matter Mater. Phys., 2012, 85, 165315.

29 L. Xia, W. Wu, Y. Hao, Y. Wang and J. Xu, Appl. Phys. Lett., 2006, 88, 152108.

30 P. Moschkau, S. Schramm, J. Hoffmann, J. Fladerer, C. Jooss, L. Wu and Y. Zhu, Mater. Sci. Eng., B, 2007, 144, 78.

31 M. Scherff, J. Hoffmann, B. Meyer, T. Danz and C. Jooss, New J. Phys., 2013, 15, 103008.

32 O. Breitenstein, J. P. Rakotoniaina, M. H. Al Rifai and M. Werner, Prog. Photovoltaics, 2004, 12, 529.

33 Y. Taur and T. H. Ning, Fundamentals of Modern VLSI Devices, Cambridge University Press, 2nd edn., 2009, pp. 125-126.

34 P. M. Solomon, J. Jopling, D. J. Frank, C. D'Emic, O. Dokumaci, P. Ronsheim and W. E. Haensch, J. Appl. Phys., 2004, 95, 5800.

35 K. J. B. M. Nieuwesteeg, M. van der Veen, T. J. Vink and J. M. Shannon, J. Appl. Phys., 1993, 74, 2581.

36 W. Shockley and W. T. Read, Phys. Rev., 1952, 87, 835.

37 C. Sah, R. N. Noyce and W. Shockley, Proc. IRE, 1957, 45, 1228.

38 A. Schenk and U. Krumbein, J. Appl. Phys., 1995, 78, 3185.

39 U. Rau, Appl. Phys. Lett., 1999, 74, 111.

40 V. Nadenau, U. Rau, A. Jasenek and H. W. Schock, J. Appl. Phys., 2000, 87, 584.

41 A. R. Riben and D. L. Feucht, Int. J. Electron., 1966, 20, 583. 42 M. Brötzmann, U. Vetter and H. Hofsäss, J. Appl. Phys., 2009, 106, 063704.

43 H. Hofsäss (II. Physikalisches Institut Universität Göttingen), Private communication. The simulations are performed with the SDTrimSP software (Eckstein et al., MPI for Plasma Physics - IPP Report12/8, 2011) and take into account the precise geometry of the FIB preparation process.

44 E. Montoya, S. Bals, M. D. Rossell, D. Schryvers and G. Van Tendeloo, Microsc. Res. Tech., 2007, 70, 1060.

45 N. I. Kato, J. Electron Microsc., 2004, 53, 451.

46 Y. Yabuuchi, S. Tametou, T. Okano, S. Inazato, S. Sadayama, Y. Yamamoto, K. Iwasaki and Y. Sugiyama, J. Electron Microsc., 2004, 53, 471.

47 S. Dong, R. Yu, S. Yunoki, J.-M. Liu and E. Dagotto, Phys. Rev. B: Condens. Matter Mater. Phys., 2008, 78, 064414.

48 S. S. Rao, S. Tripathi, D. Pandey and S. V. Bhat, Phys. Rev. B: Condens. Matter Mater. Phys., 2006, 74, 144416.

49 C. L. Lu, S. Dong, K. F. Wang, F. Gao, P. L. Li, L. Y. Lv and J.-M. Liu, Appl. Phys. Lett., 2007, 91, 032502.

50 A. Sadhu, T. Kramer, A. Datta, S. A. Wiedigen, J. Norpoth, C. Jooss and S. Bhattacharyya, Chem. Mater., 2012, 24, 3758.

$51 \mathrm{M}$. Feuchter and M. Kamlah, (Forschungszentrum Karlsruhe), Private communication. Details will be published elsewhere. 
52 I. Denk, W. Münch and J. Maier, J. Am. Ceram. Soc., 1995, 78, 3265.

53 V. A. Gasparov, S. N. Ermolov, G. K. Strukova, N. S. Sidorov, S. S. Khassanov, H.-S. Wang, M. Schneider, E. Glaser and W. Richter, Phys. Rev. B: Condens. Matter Mater. Phys, 2001, 63, 174512.

54 K. S. Takahashi and H. Y. Hwang, Appl. Phys. Lett., 2008, 93, 082112.

55 J. D. Ferguson, Y. Kim, L. Fitting Kourkoutis, A. Vodnick, A. R. Woll, D. A. Muller and J. D. Brock, Adv. Mater., 2011, 23, 1226.

56 L. C. Walters and R. E. Grace, J. Phys. Chem. Solids, 1967, 28, 245.

57 R. Waser, T. Baiatu and K.-H. Härdtl, J. Am. Ceram. Soc., 1990, 73, 1654.

58 H. B. Huntington and A. R. Grone, J. Phys. Chem. Solids, 1961, 20, 76.

59 L. Fitting Kourkoutis, H. L. Xin, T. Higuchi, Y. Hotta, J. H. Lee, Y. Hikita, D. G. Schlom, H. Y. Hwang and D. A. Muller, Philos. Mag., 2010, 90, 4731.

60 F. M. F. de Groot, M. Grioni, J. C. Fuggle, J. Ghijsen, G. A. Sawatzky and H. Petersen, Phys. Rev. B: Condens. Matter Mater. Phys., 1989, 40, 5715.

61 M. P. Kocher, D. A. Muller and P. Rez, Microsc. Microanal., 2003, 9(suppl. 02), 842.

62 M. Uijttewaal and P. E. Blöchl, (Technische Universität Clausthal), Private communication. The appropriate DFT calculations for PCMO based on the PBE0r functional will be published elsewhere.

63 S. H. Jeon, B. H. Park, J. Lee, B. Lee and S. Han, Appl. Phys. Lett., 2006, 89, 042904.

64 S.-L. Li, D. S. Shang, J. Li, J. L. Gang and D. N. Zheng, J. Appl. Phys., 2009, 105, 033710.
65 S. J. Ashcroft and E. Schwarzmann, J. Chem. Soc., Faraday Trans. 1, 1972, 68, 1360.

66 G. V. Samsonov, The Oxide Handbook, Plenum Press, 1st edn, 1973.

67 Y. Nagano, J. Therm. Anal. Calorim., 2002, 69, 831.

68 G. H. Aylward and T. J. V. Findlay, SI Chemical Data, John Wiley \& Sons, 2nd edn, 1974.

69 T. Bak, J. Nowotny, M. Rekas, C. C. Sorrell and E. R. Vance, Solid State Ionics, 2000, 135, 557.

70 S. Carter, A. Selcuk, R. J. Chater, J. Kajda, J. A. Kilner and B. C. H. Steele, Solid State Ionics, 1992, 53-56, 597.

71 T. Ishigaki, S. Yamauchi, K. Kishio, J. Mizusaki and K. Fueki, J. Solid State Chem., 1988, 73, 179.

72 M. Quintero, P. Levy, A. G. Leyva and M. J. Rozenberg, Phys. Rev. Lett., 2007, 98, 116601.

73 S. Schramm, J. Hoffmann and C. Jooss, J. Phys.: Condens. Matter, 2008, 20, 395231.

74 D. Emin, Phys. Rev. B: Condens. Matter Mater. Phys., 2006, 74, 035206.

75 M. J. Rozenberg, I. H. Inoue and M. J. Sánchez, Phys. Rev. Lett., 2004, 92, 178302.

76 M. J. Rozenberg, I. H. Inoue and M. J. Sánchez, Appl. Phys. Lett., 2006, 88, 033510.

77 Z. Jirák, S. Krupička, Z. Šimša, M. Dlouhá and S. Vratislav, J. Magn. Magn. Mater., 1985, 53, 153.

78 L. Wu, R. F. Klie, Y. Zhu and C. Jooss, Phys. Rev. B: Condens. Matter Mater. Phys., 2007, 76, 174210.

79 M. V. Abrashev, J. Bäckström, L. Börjesson, M. Pissas, N. Kolev and M. N. Iliev, Phys. Rev. B: Condens. Matter Mater. Phys., 2001, 64, 144429.

80 V. Dediu, C. Ferdeghini, F. C. Matacotta, P. Nozar and G. Ruani, Phys. Rev. Lett., 2000, 84, 4489. 